\title{
Dual mobility versus unipolar total hip arthroplasty for neck of femur fractures : a single centre study
}

\author{
Zain Sadozai, Richard Limb, Syed Awais Bokhari, Aaron NG, Manjit Bhamra
}

From the Pinderfields General Hospital, Wakefield, United Kingdom

Current national guidelines (NICE) recommends that all medically fit, independently-mobile patients without cognitive impairment receive a total hip arTHAoplasty(THA) for displaced intracapsular neck of femur (NOF) fractures. Dislocation is a concern(2-10\%). Dual mobility cups have been suggested to address this complication. Our study sets out to compare dislocation rates between dual mobility cups versus unipolar cups.

We performed a retrospective single centre multiple surgeon study of all THAs performed for NOFs between January 2012 and May 2018. A total of 322 total hip replacements (127 dual mobility and 195 unipolar; Age range of patients, 29 to 91, mean 70 years) were identified for analysis using a database. Data was obtained from electronic patient records and radiographs.

12 patients sustained a dislocation of their THA out of our 322 patients. Of these, 10 dislocations occurred in the unipolar group $(5.13 \%)$. From the dual mobility cups, 2 had dislocations( $1.57 \%$ ), both with a $28 \mathrm{~mm}$ head. Both of these dislocations were in alcohol dependent patients with increased susceptibility to falls. Statistical analysis of our data was performed using chi-squared test $(p$ value $=0.0723$ )

In 'Getting It Right First Time' (GIRFT), the authors recommend that all patients that sustain a NOF fracture meeting the criteria of a THA to be offered a dual mobility acetabular cup to reduce the risk of dislocation. The cost of the dual mobility acetabular cup is offset from the cost of overall revision surgery. Limitations of our study are its retrospective nature and selection bias.

No benefits or funds were received in support of this study. None of the authors have a conflict of interest.
Keywords : Dual mobility ; fracture femur ; dislocation ; comparison; hip arthroplasty.

\section{INTRODUCTION}

Total Hip Arthropasty (THA) is a recognised and established treatment for end stage osteoarthritis, however it is not without potential complications. Dislocation of the articulation is uncommon but remains problematic even in the hands of skilled surgeons. THA dislocation rates range from $1.1-5.8 \%$, making it the second most common complication encountered after infection $(1,2,3,4,5,6)$. Furthermore, the rates of dislocations have been found to be higher when THA is used in the management of fractured neck of femur (NOF) relative to primary arthroplasty, compounding the physical and physiological insult this injury carries (7).

Zain Sadozai, MRCS

Richard Limb, MRCS

Syed Awais Bokhari, FRCS (Orth)

Aaron NG, FRCS (Orth)

- Manjit Bhamra, FRCS (Orth)

Trauma and Orthopaedic Department, Pinderfields General Hospital, Wakefield, United Kingdom.

Correspondence : Mr Zain Sadozai, 102 Simpsons Apartments, Haworth Close, Halifax, HX1 2NJ, United Kingdom.

Email : zainsadozai@gmail.com

- 2021, Acta Orthopædica Belgica. 
Dual Mobility Total Hip Arthroplasty (DM-THA) has been developed to address this issue. DMTHA was first developed in France by Professor Gilles Bousquet and then engineered by Andre Rambert in 1974 (8). They managed to combine Sir John Charnley's principle of low friction, low torque arthroplasty and McKee-Farrar's concept of utilising large diameter femoral heads, to increase the head neck ratio, therefore reducing dislocations. The cup in these prostheses consists of two components; a polyethylene hemisphere that sits within the smooth metal acetabular shell. Within this construct sits the femoral head, providing two articulating surfaces within the arthroplasty. The primary movement occurs at the inner bearing between the femoral head and polyethylene liner. The secondary movement occurs between the polyethylene liner and the acetabulum shell outside the normal range of movement. These articulations allow a greater range of movement prior to component impingement, thereby reducing the risk of dislocation (7). Improvements in DMTHA over the years have produced prostheses with reduced rates of volumetric polyethylene wear and intraprosthetic dislocation when compared to earlier versions $(9,10)$. This has led to an increase use in primary arthroplasty with successful reductions in dislocation rates (11). The question we sought to answer was whether the use of bi-articular cups in the case of NOF fracture management will similarly lead to reduced dislocation rates (12). This question forms the rationale for this study, as if proven both clinically and cost effective, the use of DM-THA could be supported in cases that are inherently high risk for dislocation.

\section{METHODS}

This retrospective single centre cohort study looked at consecutive THA cases performed between January 2012 and May 2018 in a busy Trauma and Elective Orthopaedic Unit in the United Kingdom.

Our inclusion criteria was all patients who underwent THA for a displaced intracapsular NOF fracture. Pathological fractures were excluded with the exception of fragility fractures. Selection to undertake dual-mobility cup replacement due to high risk of dislocation as well as the use of cement was the operating surgeon's clinical decision. A 6 to 8 week prospective routine follow-up was performed following surgery. Patients were asked in regards to the occurrence of any complications following surgery. Furthermore, all individual patient records were searched until July 31, 2018 to find information about any dislocations.

Recorded data covered a total of 328 cases and included the following: Patient demographics (including age and gender), prosthesis used (including head/cup diameters and cup type), and complications including dislocations, re-dislocation frequency and revision procedures. The mean follow-up time was 37 months.

Statistical analysis was conducted using Microsoft Excel software. Frequencies, means and proportions were used to describe the data. A chisquared test was used to compare proportions of our results. Confidence intervals are generated to the 95\% level.

\section{RESULTS}

Out of the initial 328 patients, 6 were lost to follow-up. There were 127 DM-THA in our cohort of 322 patients undergoing THA. The mean number of THA performed for NOF fractures was 55 per year. Femoral head size in the dual mobility acetabular articulation consisted of 22 patients with $22 \mathrm{~mm}$ head size (17\%), 104 patients with $28 \mathrm{~mm}$ head size $(82 \%)$ and 1 patient with a $32 \mathrm{~mm}$ head size (1\%). In comparison, in the conventional THA group there were 2 patients with the $22 \mathrm{~mm}$ head size (1\%), 107 patients with the $28 \mathrm{~mm}$ head size $(55 \%)$, 53 patients with a $32 \mathrm{~mm}$ head size $(27 \%)$ and 33 patients with the larger $36 \mathrm{~mm}$ head size $(17 \%)$. The unipolar THA group consisted of 58 cemented, 28 hybrid and 109 uncemented prosthesis and the DMTHA consisted of 41 cemented, 9 hybrid and 62 uncemented prosthesis.

12 patients sustained a dislocation of their THA in our cohort of 322 patients. Of these, 10 dislocations occurred in the unipolar group $(5.13 \%)$, 7 of which were with $28 \mathrm{~mm}$ femoral head sizes and the remaining 3 dislocations were from the group with a femoral head size of $32 \mathrm{~mm}$. There were no 
dislocations from the group with $36 \mathrm{~mm}$ femoral head size. From the dual mobility acetabular articulations, 2 dislocations were observed (1.57\%). Both of these dislocations were within the $28 \mathrm{~mm}$ head size group. In the unipolar cup group, 5 patients had one dislocation, 3 patients had two dislocations and one patient each who had 4 and 5 dislocations respectively. There was 1 patient in the dual mobility cup group who had one dislocation, and the other patient had two. Both of these dislocations were in alcohol dependent patients with increased susceptibility to falls. Statistical analysis performed for the rate of dislocations between the two groups using the chi-squared test produced a $\mathrm{p}$-value of 0.0723 .

\section{DISCUSSION}

Stability is a concern in THA, with dislocation being one of the most common complications of hip arthroplasty. According to the Australian National Joint Replacement Registry, dislocation is the second most common cause of revision arthroplasty and the most common cause for subsequent revision procedures (13). An overall dislocation rate of $6 \%$ for conventional THA for fractures of the femoral neck is reported in a prospective cohort study of 713 consecutive hips (14). Dislocation following THA is also associated with significant patient morbidity (15). Recently, there has been an increase in the utilisation of DM-THA for patients who are at high risk of dislocation (16). The main patient related risk factors for increased risk of dislocation include : elderly patient group ; prior hip surgery ; American Society of Anaesthesiologists (ASA) grade $>3$; neuromuscular disease ; and body mass index (BMI) of $30 \mathrm{~kg} / \mathrm{m}^{2}$ or greater, preoperative diagnosis of avascular necrosis, inflammatory arthritis or fractured NOF (17). Our study investigates displaced NOF fractures which are known to have a higher rate of dislocation than THA performed for primary osteoarthritis (7). A dual effect is present as NOF fracture patients following a traumatic injury usually also, on average, have a higher ASA grade than patients undergoing primary THA for osteoarthritis. One study examining 2023 patients found the rate of dislocation to be nearly ten times greater in ASA 4 patients when compared to those ASA 1 (18). Implants which can reduce dislocation rates in this high-risk subgroup would be critical in improving patient outcomes. Our study demonstrates approximately threefold decrease in dislocation rates when DM-THA are used for NOF fracture patients in comparison to a unipolar THA system. We observed no dislocations in any of the dual mobility cups which were using the $32 \mathrm{~mm}$ diameter femoral head size. This potential improved stability from using a larger diameter femoral head size may be explained due to the increase in jump distance and head neck ratio.

Our results are supported by current literature. A large meta-analysis of dual mobility articulation showed a mean dislocation rate of $0.9 \%$ in primary THA and 3.0\% when used in revision THA (19). The French society of Orthopaedic Surgery and Traumatology reported a $1.4 \%$ rate of dislocation when using dual mobility cups for fractures of the femoral neck in the elderly (20). THA for femoral neck fracture in the elderly population is associated with higher rates of dislocations $(8 \%$ $\sim 11 \%$ ) even though THA shows better clinical and functional scores $(21,12)$. One study suggests that DM-THA is more favourable when compared to hemiarthroplasty for this patient group, however further research is required into knowing the indications and cost effectiveness of such an intervention (12) Other comparative clinical studies report less favourable results with dislocation rates similar between DM-THA and hemiarthroplasty $(22,23)$. The advantages of DM-THA specifically when compared to unipolar THA is not well known in current literature; although some early studies have highlighted potential benefits, further work is required to support this (12).

Prosthesis dislocation has significant cost implications for patient management, and dual mobility systems lower the risk of dislocations and subsequent revision surgery as mentioned. Their routine use may not be cost effective, however it is necessary to understand its efficacy when used in specific high risk groups such as those with femoral neck fractures. The cost of managing a dislocated THA is more than three times that of an uncomplicated procedure (24). A study used decision analysis 
software to evaluate the cost effectiveness of DMTHA in a group of patients with spinal deformities who were at high risk of dislocation. They found the more expensive DM-THA to be cost saving under the base case conditions (25). Research into the cost effectiveness of introducing DM-THA in a French healthcare system has also been published; a database of 80,405 patients who underwent a THA with a four year follow up. Total costs of all resources used in their management including those for any future dislocations were calculated. Their results revealed DM-THA had 3283 fewer dislocations per 100,000 patients. A markov model for cost effectiveness suggested DM-THA had substantial cost savings compared to a fixed bearing arthroplasty (26).

Another cost utility model investigating the cost effectiveness of dual mobility implants for primary THA found them to be cost saving for even routine arthroplasty use as long as they met specific economic and clinical benchmarks such as not exceeding the implant cost for more than $\$ 1023$ than the conventional implants (27). A Cost utility analysis study by the authors a year later examining direct and indirect costs were that DM-THA exhibited absolute dominance over conventional THA with lower accrued costs and higher accrued utility showing clear cost effectiveness from a societal perspective for DM-THA (28). This evidence supports that not only can dual mobility articulations improve risk of dislocations in an already high risk vulnerable group but can prove to be a highly cost effectiveness implementation when applied with a careful managerial strategy.

The presence of a thin polyethylene liner in-between two articulating surfaces has been theorised to predispose such a prosthesis to increased volumetric polyethylene wear; this may have contributed to early failure and intraprosthetic dislocations seen in earlier models. Modern models do not seem to exhibit the same susceptibility ; design updates with highly crossed linked polyethylene, the use of a sub-hemispherical shell and optimized head neck ratio appear to have eliminated this issue $(9,10)$. A cement - metal interface in dual mobility articulations could result in an insufficient cement mantle predisposing to cement fractures. Elevated metal ion levels have been associated with the use of a dual mobility cobalt chromium liner and risk of revision due to adverse local tissue reaction (ALTR) to metal. However clinical studies have reported no patients with elevated cobalt levels, including those patients that had been revised due to apparent Adverse Local Tissue Reaction at four year follow up. No clinical effect is seen and therefore use of DM-THA is supported (29).

Limitations of our study are its retrospective nature and selection bias. However we felt this was necessary in order to obtain high number of patients for our study.

\section{CONCLUSION}

In the mantra of 'Getting It Right First Time' (GIRFT), we recommend that all patients that sustain a femoral neck fracture meeting the criteria of a total hip replacement to be offered a dual mobility acetabular cup to reduce the risk of dislocation. We find in our experience that DMTHA a more successful procedure in the setting of trauma when compared to unipolar THA with a satisfactory mean follow up period. The cost of the dual mobility acetabular cup is offset from the cost of further procedures and overall revision surgery.

\section{REFERENCES}

1. Lewinnek GE, Lewis JL, Tarr R, Compere CL, Zimmerman JR. Dislocations aftertotal hip-replacement arthroplasties. J. Bone Joint Surg. [Am], 1978, Vols. 60-A : 217-220.15.

2. Meek RM, Allan DB, McPhillips G, Kerr L, Howie CR. Epidemiology of dislocation after total hip arthroplasty. Clin .Orthop. Relat. Res., 2006, Vols. 447 : 9-18.16.

3. Sanchez-Sotelo J, Berry DJ. Epidemiology of instability after total hip replace-ment. Orthop. Clin. North Am., 2001, Vols. 32 : 543-552, vii. vii. 17.

4. Woo RY, Morrey BF. Dislocations after total hip arthroplasty. J. Bone Joint Surg. [Am], 1982, Vols. 64-A : 1295-1306.

5. Boyer B, Philippot R, Geringer J, Farizon F. Primary total hip arthroplasty withdual mobility socket to prevent dislocation : a 22 -year follow-up of 240 hips. Int. Orthop., 2012, Vols. $36: 511-518.22$.

6. Combes A, Migaud H, Girard J, Duhamel A, Fessy MH. Low rate of dislocationof dual-mobility cups in primary total hip arthroplasty. Clin. Orthop. Relat. Res., 2013, Vols. $471: 3891-3900$. 
7. L. Matsen Ko, W. J. Hozack. The dual mobility cup : what problems does it solve? s.l. : The Bone \& Joint Journal, 1 Jan 2016, Vols. Vol. 98-B.

8. Bousquet GDF, Debiesse JL, Girardin P, Relave M, Israeli A. The ceramiccoated cementless total hip arthroplasty: basic concepts and surgical technique. $J$. Orthop. Surg. Tech., $1985 ; 1$ : 15-28.

9. Laura A, Hothi H, Battisti C, et al. Wear of dual-mobility cups : a review article. s.1. : Int. Orthop., 2017 ; 41 : 625633.

10. Vielpeau C, Lebel B, Ardouin L, Burdin G, Lautridou C. The dual mobility socketconcept : experience with 668 cases. Int. Orthop., 2011, Vols. 35 : 225-230.

11. Romagnoli M, Grassi A, Costa GG, Lazaro LE, Lo Presti M, Zaffagnini S. The efficacy of dual-mobility cup in preventing dislocation after total hip arthroplasty: a systematic review and meta-analysis of comparative studies. Int. Orthop. 2019 May ; 43(5) : 1071-1082. Epub 2018 Jul 21 .

12. K.J. Oh, D.K. Lee, S.Y. Ki. Can total hip arthroplasty using mobility cup be a wise option for displaced femoral neck fractures in patients aged older than 70 years? Prospective cohort multicentre study and systemic review. The Bone \& Joint Journal, Feb 2018, Vols. 99-B.

13. Australian Orthopaedic Association. National Joint Replacement Registry. Hip and Knee Arthroplasty, 2016, Vol. https:/aoanjrr.sahmri.com/documents/10180/217745/ Hip and Knee Arthroplasty.

14. Anders Enocson, Carl-Johan Hedbeck, Jan Tidermark, Hans Pettersson, Sari Ponzer, Lasse J Lapidus. Dislocation of total hip replacement in patients with fractures of the femoral neck. A prospective cohort study of 713 consecutive hips. Acta Orthop. 2009 Apr 29 ; 80(2) : 184-189.

15. Abdel MP, Cross MB, Yasen AT, Haddad FS. The functional and financial impactof isolated and recurrent dislocation after total hip arthroplasty. Bone Joint J., 2015, Vols. 97-B : 1046-1049.

16. Farizon F, de Lavison R, Azoulai JJ, Bousquet G. Results with a cementless alumina-coated cup with dual mobility. A twelve-year follow-up study. Int. Orthop., 1998, Vols. 22 : 219-224.

17. C. W. Jones, I. De Martino, R. D'Apolito, A. A. Nocon, P. K. Sculco, T. P. Sculco. The use of dual-mobility bearings in patients at high risk of dislocation. The Bone \& Joint Journal, 16 Jan 2019, Vols. 101-B.

18. Jolles BM, Zangger P, Leyvraz PF. Factors predisposing to dislocation after primary total hip arthroplasty: a multivariate analysis. J. Arthroplasty, 2002, Vols. 17 : 282288.

19. I. De Martino, R. D'Apolito, V. G. Soranoglou, L. A. Poultsides, P. K. Sculco, T. P. Sculco. Dislocation following total hip arthroplasty using dual mobility acetabular components : a systematic review. The Bone \& Joint Journal, 1 Jan 2017, Vols. 99-B.
20. Adam P, Philippe R, Ehlinger M, Roche O, Bonnomet F, Molé D, Fessy MH and (SoFCOT), French Society of Orthopaedic Surgery and Traumatology. Dual mobility cups hip arthroplasty as a treatment for displaced fracture of the femoral neck in the elderly. A prospective, systematic, multicenter study with specific focus on postoperative dislocation. Orthop. Traumatol. Surg. Res., 2012 May, Vols. 98(3) : 296-300.

21. Nich C, Vandenbussche E, Augereau B, Sadaka J. Do Dual-Mobility Cups Reduce the Risk of Dislocation in Total Hip Arthroplasty for Fractured Neck of Femur in Patients Aged Older Than 75 Years? J. Arthroplasty, 2016 Jun, Vols. 31(6) : 1256-1260.

22. Kim YT, Yoo JH, Kim MK, Kim S, Hwang J. Dual mobility hip arthroplasty provides better outcomes compared to hemiarthroplasty for displaced femoral neck fractures : a retrospective comparative clinical study. Int. Orthop., 2018 Jun, Vols. 42(6) : 1241-1246.

23. Boukebous B, Boutroux P, Zahi R, Azmy C, Guillon P. Comparison of dual mobility total hip arthroplasty and bipolar arthroplasty for femoral neck fractures: A retrospective case-control study of 199 hips. Orthop. Traumatol. Surg. Res., 2018 May, Vols. 104(3) : 369-375.

24. de Palma L, Procaccini R, Soccetti A, Marinelli M. Hospital cost of treating early dislocation following hip arthroplasty. Hip Int., 2012, Vols. 22 : 62-67.

25. A. M. Elbuluk, J. Slover, A. A. Anoushiravani, R. Schwarzkopf, N. Eftekhary, J. M. Vigdorchik. The cost-effectiveness of dual mobility in a spinal deformity population with high risk of dislocation : a computer-based model. The Bone \& Joint Journal, Oct 2018, Vols. 100-B.

26. Epinette JA, Lafuma A, Robert J, Doz M. Cost effectiveness model comparingdual-mobility to fixedbearing designs for total hip replacement in France. Orthop. Traumatol. Surg. Res., 2016, Vols. 102 : 143-148.

27. Barlow BT, McLawhorn AS, Westrich GH. The CostEffectiveness of Dual Mobility Implants for Primary Total Hip Arthroplasty : A Computer-Based Cost-Utility Model. J. Bone Joint Surg. Am., 2017 May, Vols. 3 ;99(9) : 768777.

28. B. Barlow, A. Mclawhorn, G.H. Westrich. Dual mobility implants are cost-saving for primary THA : A cost-utility analysis using direct and indirect costs. The Bone \& Joint Journal, 2018 Feb, Vols. 98-B.

29. B. P. Chalmers, D. G. Mangold, A. D. Hanssen, M. W. Pagnano, R. T. Trousdale, M. P. Abdel. Uniformly low serum cobalt levels after modular dual-mobility total hip arthroplasties with ceramic heads : a prospective study in high-risk patients. The Bone \& Joint Journal, May 2019, Vols. 101-B.

30. Farizon F, de Lavison R, Azoulai JJ, Bousquet G. Results with a cementless alumina-coated cup with dual mobility. A twelve-year follow-up study. Int. Orthop., 1998, Vols. 22 : 219-224. 